\title{
Possible role for TRPV1 in neomycin-induced inhibition of visceral hypersensitivity in rat
}

\author{
R. M. VAN DEN WIJNGAARD, ${ }^{\star}$ O. Welting, ${ }^{\star}$ D. C. BUlmer, $\dagger$ M. M. WOUTERs, $\ddagger$ K. LeE, $\dagger$ W. J. DE JONGE ${ }^{\star} \&$ \\ G. E. BOECKXSTAENS ${ }^{\star}$, \\ ^Division of Gastroenterology and Hepatology, Academic Medical Center, Amsterdam, The Netherlands \\ $\dagger$ Immuno-Inflammation CEDD, GlaxoSmithKline, Stevenage, United Kingdom \\ $\ddagger$ Division of Gastroenterology, University Hospital Gasthuisberg, Catholic University of Leuven, Leuven, Belgium
}

\begin{abstract}
Transient receptor ion channel 1 (TRPV1) is a nociceptor involved in visceral hypersensitivity. Aminoglycosides like neomycin are not only potent antibiotics but in vitro data suggest that neomycin also acts as a TRPV1-antagonist and alleviates somatic pain responses. To what extent neomycin reduces visceral hypersensitivity remains unknown. Therefore, we aimed to investigate whether neomycin can inhibit in vivo TRPV1-dependent hypersensitivity responses in two rat models of visceral pain. In the first model rats were pretreated with intraperitoneal (i.p.) capsazepine, the selective TRPV1 antagonist $S B-705498$, neomycin or vehicle alone and $30 \mathrm{~min}$ later instilled with intracolonic TRPV1-activating capsaicin. Likewise, rats were pretreated with 10 days oral neomycin and then subjected to intracolonic capsaicin. The visceromotor response (VMR) to distension was measured before and after capsaicin application. In addition, the VMR to distension was measured in adult maternal separated rats before and after acute stress. Before the 2nd distension protocol these rats were treated with i.p. neomycin, amoxycillin or vehicle alone. Our results showed that capsaicin administration induced an enhanced VMR to distension that was prevented by i.p. capsazepine, SB-705498 and neomycin. Oral neomycin treatment changed bacterial faecal content but could not inhibit capsaicin induced visceral hypersensitivity. In maternal separated rats acute stress induced an enhanced response to distension that was reversed by i.p.
\end{abstract}

Address for correspondence

R. M. van den Wijngaard, Department of Gastroenterology and Hepatology, Academic Medical Center (Room C2-310), Meibergdreef 9, 1105 AZ, Amsterdam, The Netherlands.

Tel: +31 20 5668708; fax: +31 20 6917033;

e-mail: R.vandenWijngaard@AMC.UVA.NL

Received: 27 August 2008

Accepted for publication: 26 January 2009 neomycin, but not amoxycillin. These data indicate that (i.p.) neomycin can inhibit visceral hypersensitivity to distension in a nonbactericidal manner and suggest that TRPV1-modulation may be involved.

Keywords in vivo, neomycin, rat, transient receptor ion channel 1, visceral hypersensitivity.

Abbreviations: AUC, area under curve; CFU, colony forming units; IBS, irritable bowel syndrome; i.p., intra peritoneal; MS180, 180 minutes daily maternal separation; $\mathrm{NH}$, nonhandled; SIBO, small intestinal bacterial overgrowth; TRPV1, transient receptor ion channel 1; VMR, visceromotor response; WA, water avoidance.

\section{INTRODUCTION}

Aminoglycosides like neomycin and gentamycin are commonly used in the treatment of serious infections caused by aerobic Gram-negative bacilli. Interestingly, data obtained in different rodent models suggested that these antibiotics are also potent analgesics. ${ }^{1-3}$ In mice, intracerebroventricular administration of neomycin produced dose-dependent antinociceptive activity in hot plate and tail flick tests. ${ }^{1}$ Similar results were reported for a rat model of incisional pain were intrathecal neomycin administration reduced incisional allodynia. ${ }^{2}$ Blenk et al. showed that not only central but also peripheral administration of neomycin can be effective. ${ }^{3}$ Transection of the left ventral ramus of the L5 spinal nerve led to mechanical allodynia which was prevented by local application of neomycin immediately after cutting the nerve. Although these reports clearly showed the analgesic potential of neomycin, the possible mechanism of action was not addressed. In vitro investigations however suggested that neomycin is a TRPV1 antagonist.

Transient receptor ion channel 1 is a sensoryneuron-expressed, nonselective, ligand-gated cation 
channel essential for several modalities of pain sensation. It can be activated by a number of different stimuli including noxious temperature, extracellular low $\mathrm{PH}$ and the nonphysiological stimulus capsaicin. ${ }^{4}$ Using whole-cell patch-clamp recordings of acutely isolated rat DRG neurons Zhou et al. were the first to show that neomycin inhibits capsaicin-induced currents. ${ }^{5}$ Later results, obtained with cloned TRPV1 heterologously expressed in Xenopus oocytes, suggested that neomycin inhibits TRPV1 by allosteric binding and altering channel gating. ${ }^{6}$ Whether TRPV1 is not only an in vitro but also an in vivo target of neomycin remains to be investigated. Another unanswered question is whether neomycin-induced analgesia is restricted to somatic pain alone or also effective in visceral pain models. We chose to investigate these issues in two TRPV1 dependent rat models of colonic hypersensitivity.

\section{MATERIAL AND METHODS}

All experimental protocols were approved by the Ethical Animal Research Committee of the University of Amsterdam.

\begin{abstract}
Animals
Long-Evans rats (Harlan, Horst, The Netherlands) were housed at the animal facility of the Academic Medical Center (Amsterdam, The Netherlands) under conditions of controlled light (06:00$18.00 \mathrm{~h})$, temperature $\left(20-22{ }^{\circ} \mathrm{C}\right)$ and humidity $(45 \%)$. Water and food (SDS; Technilab BMI, Someren, The Netherlands) were available ad libitum. Rats used for the maternal separation protocol (nonhandled ( $\mathrm{NH}$ ) and maternal separated animals) were bred in our own animal facilities. Nonhandled rats used for the intra-colonic capsaicin experiments were directly obtained from Harlan. Vehicle was always investigated in separate groups. All experiments were carried out with male animals only and none of the animals were used in more than one experiment.
\end{abstract}

\section{Colonic distension protocol}

Colonic distensions were performed with a latex balloon (Ultracover 8F, International Medical Products BV, Zutphen, The Netherlands) and carried out as described previously. ${ }^{7}$

Distensions in maternal separated rats (see Model 2: neonatal maternal separation and water avoidance (WA) for description of the separation model) were performed at the minimum age of 3 months and achieved by inflation of graded volumes of water $(1.0,1.5$ and $2.0 \mathrm{~mL})$ into the balloon. Length and diameter of the balloon during a $2 \mathrm{~mL}$ maximum volume distension were 18 and $15 \mathrm{~mm}$ respectively. After each $20 \mathrm{~s}$ distension period the volume of water was quickly removed and an $80 \mathrm{~s}$ resting period was introduced. Animals who received intracolonic capsaicin (see Experimental models) were studied at 2 months of age (to economize costs of purchase and housing) and subjected to lower distension volumes; $0.8,1.2$ and $1.6 \mathrm{~mL}$.

\section{Measurement of the visceromotor response (VMR) in freely moving rats and EMG data analysis}

We used a telemetric transmitter (Physiotel Implant TA10AEF20; Data Sciences International (DSI), St Paul, MN, USA) positioned in the right side of the abdominal cavity to record the EMG signals from two connected electrodes placed in the abdominal muscles. During distension protocols, animals were placed in a standard macralon cage that was positioned on top of a flat receiver with an equal surface area (DSI). The receiver was linked to a Biopac MP100 data acquisition system (Biopac Systems Inc., Santa Barbara, CA, USA) and a personal computer via a raw data analog converter (Data Sciences International). Data were acquired with AcqKnowledge software (Biopac Systems Inc., Santa Barbara, CA, USA) and analyzed as described before. ${ }^{7}$ Briefly, each 20 -s distension period and its preceding 20-s of baseline recording were extracted from the original raw EMG data file. After correction for movement and breathing, data were rectified and integrated. Absolute data sets were then obtained by subtracting the 20-s baseline recording from the 20-s distension result. Similar to previous studies ${ }^{7,8}$ the final results are given as normalized data sets, which were calculated from the absolute data by setting the highest prestress (or precapsaicin) value at $100 \%$.

\section{Experimental models}

Model 1: intracolonic capsaicin in NH rats After positioning of the balloon catheter the precapsaicin VMR to colonic distension was established at $t=0 \mathrm{~min}$. At $t=60 \mathrm{~min}$, just before re-positioning of the balloon catheter, capsaicin (Sigma-Aldrich, St Louis, MO, USA) was administered under short isoflurane anesthesia. Capsaicin concentration and application methodology were taken from Laird et al.; ${ }^{9}$ we first applied Vaseline (Boots Healthcare, Hilversum, The Netherlands) to the perianal area to avoid stimulation of somatic areas. Subsequently, $100 \mu \mathrm{L}(0.1 \%)$ of the TRPV1 agonist capsaicin, or vehicle alone (10\% ethanol, 10\% Tween-80, 80\% saline), was given with a fine cannula with a rounded tip inserted rectally, $2 \mathrm{~cm}$ from the anus. Animals were allowed to recover and the second distension protocol was performed at $t=90 \mathrm{~min}$.

Model 2: neonatal maternal separation and water avoidance (WA) Primiparous pregnant rats reared NH male pups; second time pregnant dams reared male pups that were subjected to the maternal separation protocol. During maternal separation dams were separated from the nest from postnatal day 2 to 14 for $3 \mathrm{~h}$ per day (MS180) as described previously. ${ }^{7}$ After weaning on postnatal day 22, rats were raised in pairs of two. Nonhandled pups were nursed normally.

In our earlier investigations we showed that acute WA-stress induces enhanced sensitivity to colonic distension in adult MS180- and not in NH rats. Stress-induced hypersensitivity was still present at $24 \mathrm{~h}$ post-WA and was not induced by sham WA-stress. ${ }^{7}$ Therefore, whenever acute-stress was applied in the present investigations, distensions and concurrent EMG recordings were performed just before and $24 \mathrm{~h}$ after $1 \mathrm{~h}$ WA during which rats were positioned on a pedestal surrounded by water. 


\section{Experimental protocols (in vivo pharmacological interventions)}

Inhibition of capsaicin-induced hypersensitivity to distension After positioning of the balloon catheter the baseline VMR to colonic distension was established at $t=0 \mathrm{~min}$. At $t=30 \mathrm{~min}$ rats were injected intraperitoneal (i.p.) with (i) the nonselective ${ }^{10,11}$ TRPV1 antagonist capsazepine $\left(10 \mathrm{mg} \mathrm{kg}^{-1}\right.$, SigmaAldrich) or vehicle alone (1 mL 5\% Tween-80, 5\% EtOH, 20\% DMSO, 70\% PBS), (ii) the selective TRPV1 antagonist SB-705498 ${ }^{12,13}$ (30 $\mathrm{mg} \mathrm{kg}^{-1}$, Glaxo Smith Kline, Stevenage, UK) or vehicle alone $(0.5 \mathrm{~mL} \mathrm{DMSO})$, or (iii) neomycin-sulphate $\left(100 \mathrm{mg} \mathrm{kg}^{-1} \text {, Sigma-Aldrich }\right)^{14}$ or vehicle alone $(1 \mathrm{~mL} 0.9 \%$ $\mathrm{NaCl})$. At $t=60 \mathrm{~min}, 100 \mu \mathrm{L} 0.1 \%$ capsaicin was administered as mentioned in Model 1: intracolonic capsaicin in $\mathrm{NH}$ rats. Animals were allowed to recover and the second distension protocol was performed at $t=90 \mathrm{~min}$ (see also Fig. 1A).

The possible effect of neomycin-sulphate was also tested when supplemented to drinking water; $1.43 \mathrm{~g} \mathrm{~L}^{-1}$ was added from day 1 to day $10 .{ }^{15}$ At day 10 intra-colonic capsaicin was given as described above. The VMR to colonic distension was established at day 0 and two times at day 10; $30 \mathrm{~min}$ before and $30 \mathrm{~min}$ after capsaicin treatment. In addition, the efficacy of neomycin treatment was established by the culture of aerobic and anaerobic bacteria from faecal pellets collected at day 0,2 and 10. Pellets were homogenized in sterile saline and colony forming units (CFU) were determined from serial dilutions of these homogenates plated on blood agar plates and incubated in $37^{\circ} \mathrm{C}$. Plates were evaluated after 4 (for anaerobic) or 2 (for aerobic) days of cultivation. Anaerobic bacteria were cultivated in a GasPak ${ }^{\mathrm{TM}} \mathrm{EZ}$ anaerobe container system (BD, Breda, The Netherlands) in the presence of a control culture (Pseudomonas aeruginosa, which only grows under aerobic conditions).

Inhibition of WA-induced hypersensitivity to distension in MS180 rats We have shown that capsazepine ${ }^{16}$ and SB705498 (unpublished observation) are able to reverse WA-induced visceral hypersensitivity in MS180 rats. Here, NH and MS180 rats received an i.p. injection of (i) neomycin-sulphate $\left(100 \mathrm{mg} \mathrm{kg}^{-1}\right.$, Sigma-Aldrich) or vehicle alone, or (ii) the penicillin amoxicillin (Clamoxyl $100 \mathrm{mg} \mathrm{kg}^{-1}$, Glaxo Smith Kline, Zeist,
The Netherlands) or vehicle alone $(0.9 \% \mathrm{NaCl}) 30 \mathrm{~min}$ prior to the $24 \mathrm{~h}$ post-WA time point (see also Fig. 1B). The nonaminoglycoside amoxycillin was chosen as a negative control antibiotic that does not interact with TRPV1.

\section{Compliance}

In order to anticipate possible pharmacological effects on compliance the pressure-volume relationship was determined in a subset $(n=5)$ of separated rats as published previously. ${ }^{7}$ In short, this was accomplished using a polyethylene balloon $(25-18 \mathrm{~mm})$ connected to a slightly adjusted sphygmomanometer. Measurements were performed before and after administration of the investigated compounds and the time span between measurements correlated with the experimental protocols described above.

\section{Statistical analysis}

All statistical calculations were performed using spss for windows (version 11.5.2) (SPSS Benelux BV, Gorinchem, The Netherlands). In case of EMG data, statistical significance within groups of rats for the time points before and after WA, and before and after capsaicin treatment was analyzed with the Wilcoxon signed ranks test. The test was applied for the area under the curve (AUC) of the relative response (normalized data) to colonic distension. Wilcoxon signed ranks was also used to evaluate possible compound-induced changes in compliance and the number of CFU g ${ }^{-1}$ stool before and after oral neomycin treatment.

\section{RESULTS}

\section{Intracolonic capsaicin in $\mathrm{NH}$ rats}

Colonic installation of $100 \mu \mathrm{L}$ vehicle alone did not lead to an enhanced VMR to colonic distension (dotted lines, Fig. 2A, $n=7$ ). In contrast, a significant increase in AUC was observed 30 min after intracolonic administration of $100 \mu \mathrm{L} 0.1 \%$ capsaicin (Fig. 2A, continuous
A

Figure 1 (A) Schematic representation of experimental protocols involving the inhibition of capsaicin-induced hypersensitivity to colonic distension in adult nonhandled (NH) rats. (B) Schematic representation of experiments carried out to establish the post-WA sensitivity to colonic distension in $\mathrm{NH}$ and MS180 rats when treated with antibiotics (neomycin or amoxycillin) or vehicle alone. $226 \times 164 \mathrm{~mm}(150 \times 150$ DPI).

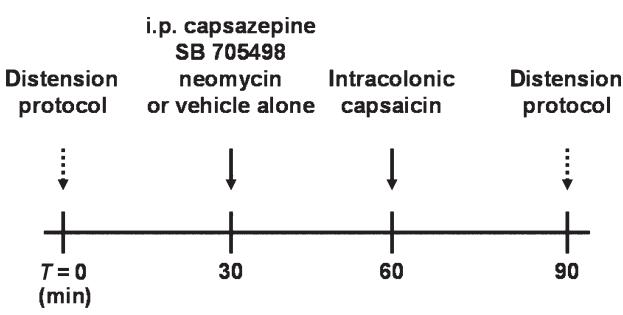

B

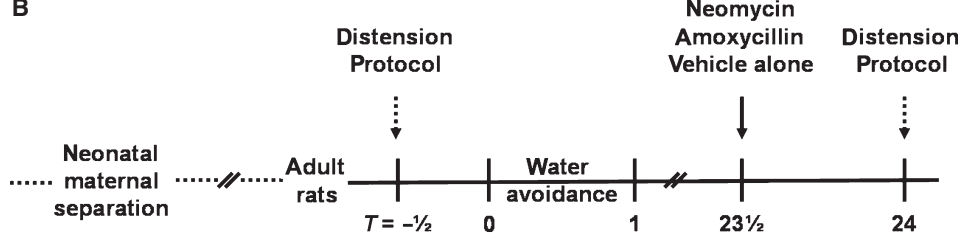

(h) 
lines, $\left.{ }^{\star} P=0.018, \quad n=7\right)$. Next, i.p. capsazepine (10 $\mathrm{mg} \mathrm{kg}^{-1}$ ) was administered $30 \mathrm{~min}$ prior to intracolonic capsaicin. This resulted in a complete inhibition of capsaicin-induced hypersensitivity to distension (continuous lines, Fig. 2B, $n=7$ ) whereas precapsaicin administration of vehicle alone could not prevent this enhanced response (dotted lines, ${ }^{\star \star} P=0.028, n=7$ ). Similar to capsazepine, the specific TRPV1-antagonist
SB-705498 (30 $\left.\mathrm{mg} \mathrm{kg}^{-1}\right)$ prevented capsaicin-induced visceral hypersensitivity (Fig. 2C, continuous lines, $n=8$, whereas vehicle alone was unable to inhibit the enhanced VMR to distension (dotted lines, ${ }^{\#} P=0.012$, $n=8$ ).

As shown in Fig. 2D, neomycin (100 mg kg-1 i.p.) is able to inhibit capsaicin-induced hypersensitivity to colonic distension when administered $30 \mathrm{~min}$ prior to
A

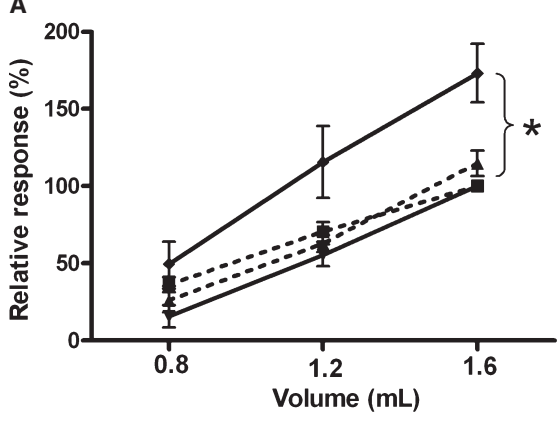

- - -pre vehicle $\rightarrow$ pre capsaicin

-\#post vehicle $\multimap$ post capsaicin

C

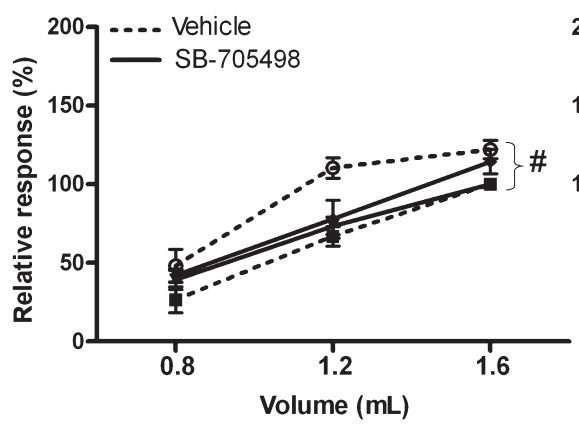

- -pre capsaicin $\rightarrow$ pre capsaicin

- $\cdots$-post capsaicin $\rightarrow$ post capsaicin

E

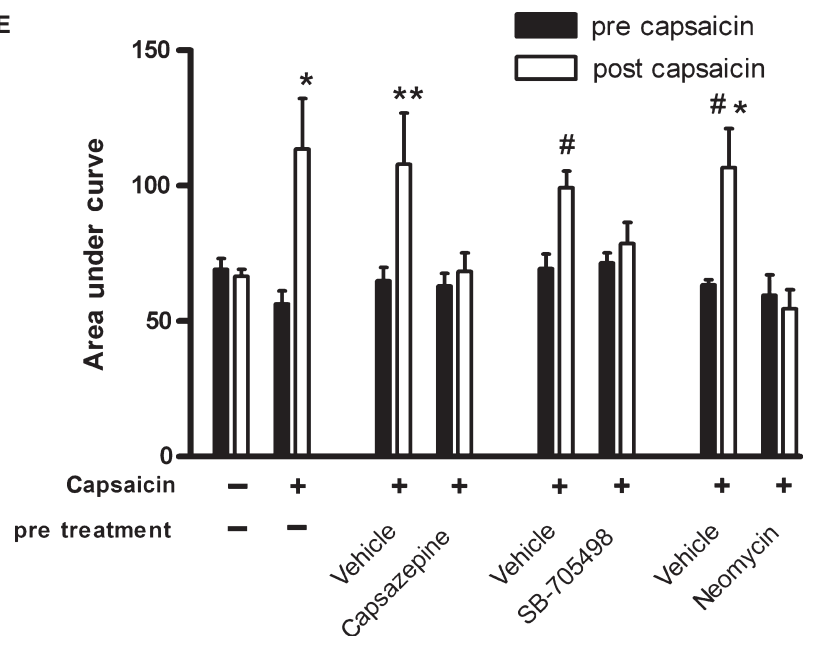

D
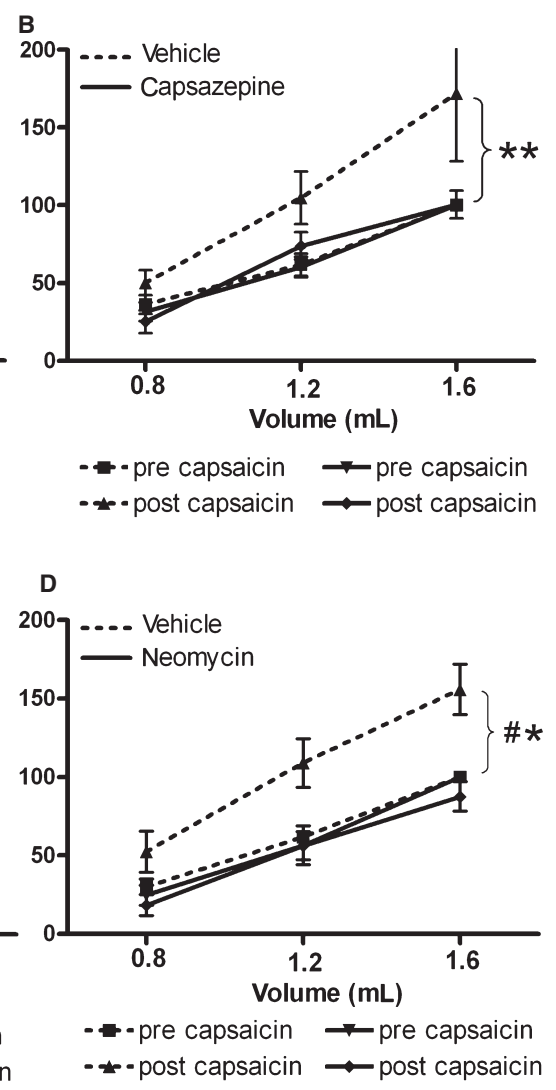

Figure 2 Effects of intracolonic capsaicin on VMR to distension in nonhandled $(\mathrm{NH})$ rats. Values are mean \pm SEM. Differences in area under the curve were evaluated for statistical significance. In contrast to vehicle alone (dotted lines), intracolonic capsaicin application induced an enhanced response to colonic distension $(\mathrm{A}$; continuous lines, prevs postcapsaicin, $\left.{ }^{\star} P=0.018\right)$. Pretreatment with nonselective TRPV1 antagonist capsazepine (continuous lines $\mathrm{B}$ ), selective TRPV1 antagonist SB-705498 (continuous lines C) or neomycin (continuous lines D) inhibited the capsaicin induced hypersensitivity to distension that was still apparent when rats were pretreated with vehicle alone (dotted lines, ${ }^{\star \star} P=0.028, P=0.012$ and $\#^{\star} P=0.028$ respectively). $3 \mathrm{E}$ shows a summary of the intracolonic capsaicin data but now depicting AUC ( $P$-values of pre- $v s$ postcapsaicin application as mentioned before). $115 \times 199 \mathrm{~mm}(600 \times 600 \mathrm{DPI})$. 
A

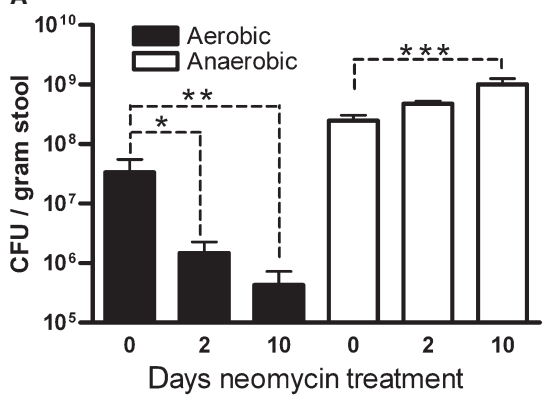

B

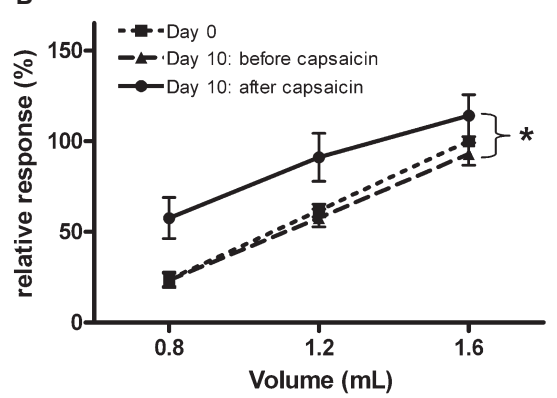

Figure 3 Effects of 10 days oral neomycin treatment in nonhandled (NH) rats. Values are mean \pm SEM. Treatment resulted in the reduction of aerobic

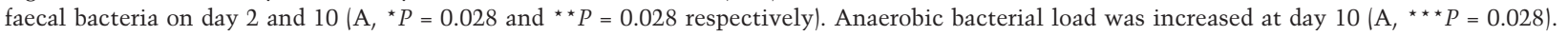
Visceromotor response (VMR) to distension was unchanged (area under curve) after 10 days of oral neomycin (B, dotted lines) and neomycin treatment was unable to prevent enhanced capsaicin-induced VMR to colonic distension at day 10 (dotted line with triangles $V s$ continuous line, ${ }^{\star} P=0.017$ ). $254 \times 104 \mathrm{~mm}(600 \times 600 \mathrm{DPI})$.

capsaicin (continuous lines, $n=7$ ). In contrast, vehicle alone is unable to prevent the enhanced response to distension (dotted lines ${ }^{\# \star} P=0.028, n=7$ ). Fig. $2 \mathrm{E}$ shows a summary of the intracolonic capsaicin data by depicting AUC: similar inhibitory results are obtained with preadministration of capsazepine, SB-705498 and neomycin .

Ten days oral neomycin treatment resulted in a 1-log reduction of aerobic faecal bacteria on day 2 and a 2-log reduction on day 10 (Fig. $3 \mathrm{~A},{ }^{\star} P=0.028$ and ${ }^{\star \star} P=0.028$ respectively). In contrast, the anaerobic bacterial load showed a 1-log increase at day 10 (Fig. 3A, ${ }^{\star \star \star} P=0.028$ ). Distension results obtained before and after 10 days oral neomycin showed no changes in VMR due to neomycin treatment per se (Fig. 3B, dotted lines, $n=10$ ). In addition, 10 days oral neomycin could not prevent the enhanced capsaicin-induced VMR to colonic distension at day $10\left({ }^{\star} P=0.017\right)$.

\section{WA-induced hypersensitivity to distension}

Water avoidance was unable to induce an enhanced response to distension in $\mathrm{NH}$ rats (Figs 4A,C, dotted lines). Similarly, when $\mathrm{NH}$ rats were treated with neomycin (Fig. 4A) or amoxycillin (Fig. 4C, continuous lines) the post-WA response to distension remained unchanged. In contrast, WA induced an enhanced VMR in vehicle treated MS180 rats (Figs 4B,D, dotted lines, ${ }^{\star} P=0.018$ and $\left.\# P=0.018\right)$. Neomycin treatment, $30 \mathrm{~min}$ prior to the post-WA distension protocol, inhibited the enhanced response to distension (Fig. 4B, continuous lines), whereas amoxycillin treated rats remained hypersensitive (Fig. 4D, continuous lines, $\#^{\star} P=0.028$ ). Fig. $4 \mathrm{E}$ shows a summary of the WA results. In this case AUC is depicted instead of relative response ( $P$-values as mentioned above).

\section{Compliance}

For each individual distension volume we compared pressures generated before and after application of the different compounds (capsaicin, capsazepine, SB-705498, neomycin or amoxicillin). We did not observe significant differences. This suggests that there are no compound induced changes in compliance (see Table 1).

\section{DISCUSSION}

Previous in vitro experiments revealed that neomycin acts as a TRPV1-antagonist. ${ }^{5,6}$ In the present study its in vivo analgesic properties were assessed in two different TRPV1-dependent rat models of visceral pain. In the first model, colonic instillation of the TRPV1agonist capsaicin led to an enhanced VMR to colonic distension. This response could be prevented by i.p. administration of capsazepine and SB-705498 (nonselective- and selective TRPV1-antagonists, respectively). Intraperitoneal administration of neomycin inhibited the capsaicin-induced response to the same extent as these compounds, suggesting that neomycin may also act as an in vivo TRPV1 antagonist.

The possibility of neomycin alleviating visceral hypersensitivity in a nonbactericidal but TRPV1dependent manner was further investigated in the rat model of maternal separation. In this model, daily separation of mother and pups during the neonatal period leads to altered behavior of the mother towards these pups and, subsequently, to an enhanced stress response at adult age. ${ }^{17}$ In adult MS180 Long-Evans rats, acute stress induces an increased VMR to colonic distension which is not observed in $\mathrm{NH}$ animals. ${ }^{7,8}$ 
A

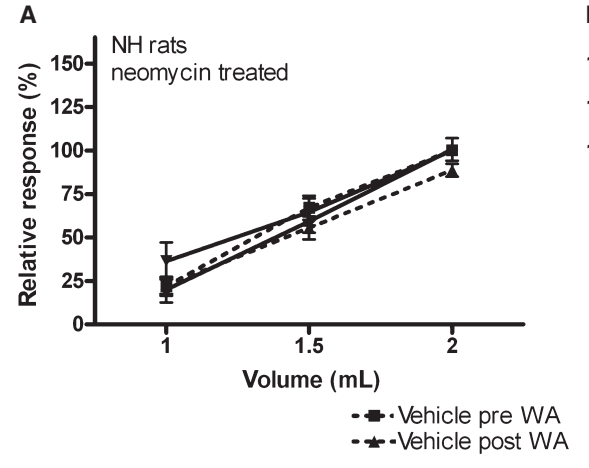

C

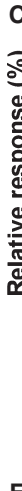

E

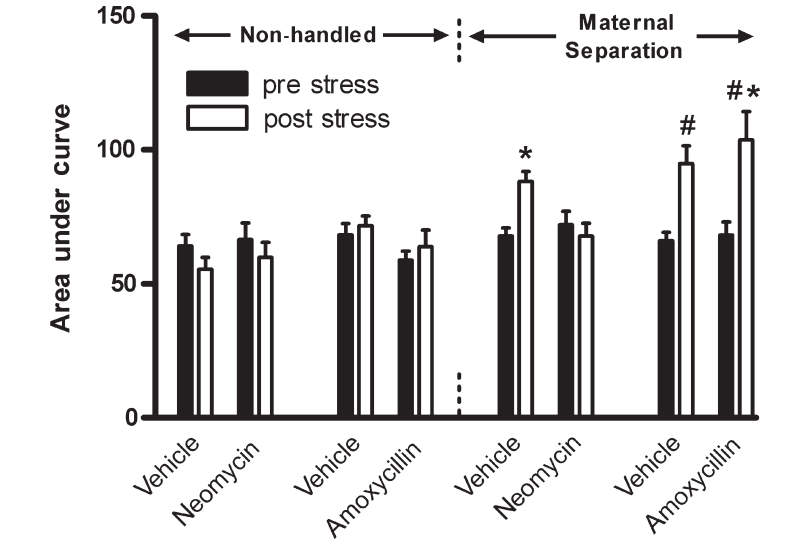

B

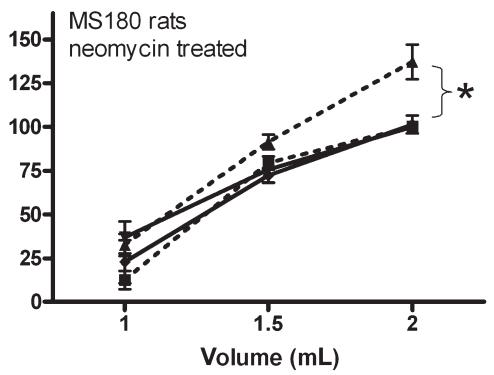

$\rightarrow$ Neomycin pre WA

Neomycin post WA

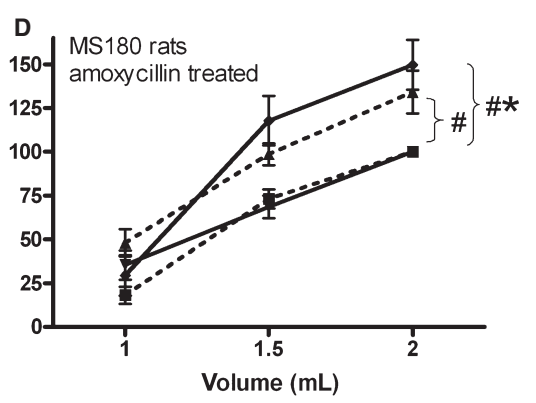

Volume $(\mathrm{mL})$

Volume $(\mathrm{mL})$

--V-Vehicle pre WA

$\rightarrow$ Amoxycillin pre WA

$\rightarrow$ Amoxycillin post WA

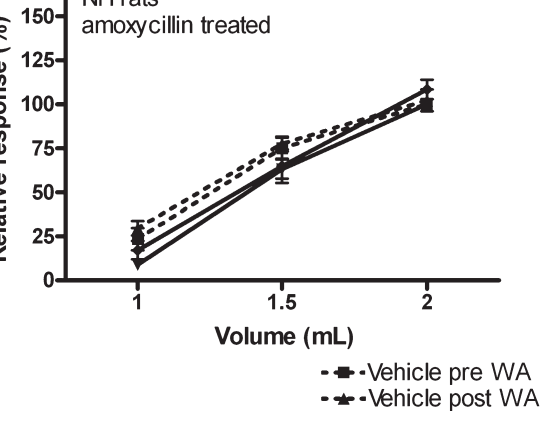

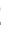

Figure 4 Effects of WA on VMR to distension in NH and MS180 rats. Values are mean \pm SEM. Differences in area under the curve were evaluated for statistical significance. WA did not induce an enhanced VMR to distension in $\mathrm{NH}$ rats $(\mathrm{A}$ and $\mathrm{C}$, dotted lines) and post-WA response to distension remained unchanged when rats were treated with neomycin $(\mathrm{A})$ or amoxycillin $(\mathrm{C}$, continuous lines). WA induced an enhanced VMR in vehicle treated MS180 rats $(B$ and $D$, dotted lines, ${ }^{\star} P=0.018$ and $P=0.018$ respectively) which was prevented by neomycin (B, continuous lines) but not amoxycillin treatment $(\mathrm{D}$, continuous lines, $\left.\#^{\star} P=0.028\right)$. E shows a summary of the results; AUC is depicted instead of relative response ( $P$-values as mentioned above). $131 \times 198 \mathrm{~mm}(600 \times 600 \mathrm{DPI})$.

Table 1 No significant differences (Wilcoxon signed ranks) were observed when comparing volume-induced pressures before and after compound application

\begin{tabular}{|c|c|c|c|c|c|c|c|c|c|c|}
\hline \multirow{3}{*}{$\begin{array}{l}\text { Distension } \\
\text { volume }(\mathrm{mL})\end{array}$} & \multicolumn{10}{|c|}{ Pressure \pm SEM $(\mathrm{mmHg})$} \\
\hline & \multicolumn{2}{|l|}{ Capsaicin } & \multicolumn{2}{|c|}{ Capsazepine } & \multicolumn{2}{|l|}{ SB-705498 } & \multicolumn{2}{|l|}{ Neomycin } & \multicolumn{2}{|c|}{ Amoxycillin } \\
\hline & Pre & Post & Pre & Post & Pre & Post & Pre & Post & Pre & Post \\
\hline 1.0 & $19.5 \pm 1.0$ & $20.5 \pm 1.0$ & $19.0 \pm 1.5$ & $18.0 \pm 1.7$ & $24.0 \pm 0.8$ & $24.0 \pm 0.8$ & $19.2 \pm 1.0$ & $20.8 \pm 1.6$ & $18.8 \pm 1.8$ & $18.3 \pm 1.3$ \\
\hline 2.0 & $35.5 \pm 2.4$ & $33.0 \pm 1.3$ & $41.6 \pm 4.7$ & $42.0 \pm 4.2$ & $37.0 \pm 1.7$ & $35.8 \pm 2.3$ & $34.8 \pm 2.3$ & $33.2 \pm 2.1$ & $33.5 \pm 3.6$ & $34.0 \pm 2.3$ \\
\hline 3.0 & $66.0 \pm 3.6$ & $69.5 \pm 5.9$ & $78.8 \pm 4.3$ & $78.0 \pm 5.0$ & $69.5 \pm 2.4$ & $70.5 \pm 2.1$ & $72.8 \pm 2.2$ & $72.4 \pm 2.8$ & $70.8 \pm 4.6$ & $69.0 \pm 5.0$ \\
\hline
\end{tabular}

Our recent data indicated that stress-induced hypersensitivity in MS180 rats depends on mast cell degranulation and subsequent TRPV1 modulation.
The latter was shown by capsazepine ${ }^{16}$ and SB-705498 (unpublished observation) mediated reversal of enhanced colonic sensitivity. In the present 
study neomycin, administered (i.p.) $30 \mathrm{~min}$ prior to the post-WA distension protocol, completely inhibited stress-induced hypersensitivity. The short time-interval between the administration of neomycin and the subsequent post-WA distension protocol makes a bactericidal effect rather unlikely. Nevertheless, to rule out this possibility, we investigated the effect of i.p. administration of the nonaminoglycoside-antibiotic amoxycillin in the same experimental set up. In contrast to neomycin, this broad spectrum penicillin was unable to reverse the stress-induced increase in VMR in MS180 rats.

Others showed that neomycin blocks capsaicin evoked membrane currents in cultured dorsal root ganglia ${ }^{5,6}$ and cloned-TRPV1 heterologously expressed in Xenopus oocytes. ${ }^{6}$ The results of the oocyte study indicated that neomycin inhibits TRPV1 activity by allosteric binding and altering channel gating. It should however be emphasized that for our in vivo studies, interactions with other ion channels or membrane receptors can not be excluded. For example, therapeutically relevant concentrations of neomycin indeed block voltage-activated $\mathrm{Ca}^{2+}$ channels. ${ }^{18}$ Whether this mechanism plays a role in the present study is unknown: neomycin has a preference for P-type $\mathrm{Ca}^{2+}$ channels and to our knowledge their role has not been investigated in any of the colonic hypersensitivity models. Neomycin also inhibits TTX-resistant $\mathrm{Na}^{+}$currents in isolated small dorsal root ganglia neurons ${ }^{19}$ and it was shown previously that colitis-induced hyper-excitability of colonic sensory neurons is associated with increased Nav1.8 currents. ${ }^{20}$ In addition, when intracolonic capsaicin was administered to Nav1.8-null mutants, significantly fewer nociceptive behaviours were observed compared to wild-type mice. ${ }^{21}$ These data suggest that the neomycin-induced reversal of capsaicin-induced hypersensitivity to distension may, in addition to TRPV1, also relate to Nav1.8 modulation. Nevertheless, in our models complete inhibition of visceral hypersensitivity was achieved by the TRPV1-selective antagonist SB-705498, suggesting that enhanced sensitivity to distension mainly depended on TRPV1.

In the present investigations we used the VMR to colorectal distension as an indirect measure for visceral hypersensitivity. Visceral hypersensitivity is thought to be an important pathophysiological mechanism in the irritable bowel syndrome (IBS). ${ }^{22}$ This functional bowel disorder is characterized by abdominal pain or discomfort associated with defecation or change in bowel habit. One of the new theories about its etiology suggests that small intestinal bacterial overgrowth (SIBO) leads to symptoms of IBS. ${ }^{23}$ When neomycin was used to eradicate possible SIBO in a placebo-controlled patient trial, ${ }^{24}$ a modest but significant reduction in global symptom or composite score was achieved. Especially because increased numbers of colonic TRPV1 ${ }^{+}$mucosal nerve fibers were reported to correlate with abdominal pain scores ${ }^{25}$ it is tempting to speculate that neomycin also targeted TRPV1 in the IBS patient study. Our current results can however not justify such a conclusion.

While aminoglycosides are poorly absorbed from the gut, the IBS patients received oral treatment. When neomycin was added to the drinking water of $\mathrm{NH}$ rats, a 10-days treatment protocol resulted in a clear shift in the bacterial composition of faecal pellets, but capsaicininduced hypersensitivity to distension could not be prevented. This negative finding suggests that, at least in rats, absorption of neomycin is not high enough to reach effective systemic concentrations. However, a direct translation to the IBS data is not possible. Firstly, it is known that the permeability of the human gut is considerably higher than that of rats. ${ }^{26}$ Secondly, gut permeability in IBS patients may be compromised ${ }^{27,28}$ and therefore lead to enhanced neomycin absorption compared with controls $(0.58 \%$ of an orally administered $2 \mathrm{~g}$ dose is absorbed in $48 \mathrm{~h}$ in normal human subjects). ${ }^{29}$ The latter makes it impossible to reliably calculate the absorbance rate of neomycin in these patients (data on serum or urine concentrations are not available). Thus, a definitive conclusion on this intriguing possibility requires further experiments in humans, which is beyond the scope of these investigations.

Earlier results indicated that neomycin is a potent analgesic in somatic pain responses. In vitro investigations suggested that this may relate to a TRPV1antagonistic effect. We report here that neomycin is also capable of reversing established visceral hypersensitivity. Moreover, our data indicate that, regarding its anti-nociceptive properties, TRPV1 is an in vivo target of neomycin. Finally, these data again show that TRPV1 is an important candidate target molecule to develop effective treatment strategies in disorders associated with visceral hypersensitivity.

\section{ACKNOWLEDGEMENTS}

RMvdW is supported by the Technology Foundation STW, Applied Division of NWO, and the Technology Program of the Ministry of Economic Affairs (NWO-STW, grant AKG 5727), WJdeJ is supported by a Marie Curie Intra-European Fellowship within the 6th European Community Framework Programme. GEB is supported by a grant (Odysseus program, grant G.0905.08) of the Flemish Scientific organization (FWO). Preliminary results of these investigations were presented at Neurogastroenterology and Motility 2006 Joint International Meeting (Boston, $\mathrm{Ma}, \mathrm{USA})^{30}$ and 2008 Joint International Meeting (Lucerne, Switzerland). ${ }^{31}$ 


\section{REFERENCES}

1 Ocana M, Baeyens JM. Analgesic effects of centrally administered aminoglycoside antibiotics in mice. Neurosci Lett 1991; 126: 67-70.

2 Prado WA, Machado Filho EB. Antinociceptive potency of aminoglycoside antibiotics and magnesium chloride: a comparative study on models of phasic and incisional pain in rats. Braz J Med Biol Res 2002; 35: 395-403.

3 Blenk KH, Habler HJ, Janig W. Neomycin and gadolinium applied to an L5 spinal nerve lesion prevent mechanical allodynia-like behaviour in rats. Pain 1997; 70: 155-65.

4 Geppetti P, Trevisani M. Activation and sensitisation of the vanilloid receptor: role in gastrointestinal inflammation and function. $B r J$ Pharmacol 2004; 141: 1313-20.

5 Zhou Y, Zhou ZS, Zhao ZQ. Neomycin blocks capsaicin-evoked responses in rat dorsal root ganglion neurons. Neurosci Lett 2001; 315: 98-102.

6 Raisinghani M, Premkumar LS. Block of native and cloned vanilloid receptor 1 (TRPV1) by aminoglycoside antibiotics. Pain 2005; 113: 123-33.

7 Welting $\mathrm{O}$, van den Wijngaard RM, de Jonge WJ, Holman R, Boeckxstaens GE. Assessment of visceral sensitivity using radio telemetry in a rat model of maternal separation. Neurogastroenterol Motil 2005; 17: 838-45.

8 Coutinho SV, Plotsky PM, Sablad M et al. Neonatal maternal separation alters stress-induced responses to viscerosomatic nociceptive stimuli in rat. Am I Physiol Gastrointest Liver Physiol 2002; 282: G307-16.

9 Laird JM, Martinez-Caro L, GarciaNicas E, Cervero F. A new model of visceral pain and referred hyperalgesia in the mouse. Pain 2001; 92: 335-42.

10 Liu L, Simon SA. Capsazepine, a vanilloid receptor antagonist, inhibits nicotinic acetylcholine receptors in rat trigeminal ganglia. Neurosci Lett 1997; 228: 29-32.

11 Docherty RJ, Yeats JC, Piper AS. Capsazepine block of voltage-activated calcium channels in adult rat dorsal root ganglion neurones in culture. Br I Pharmacol 1997; 121: 1461-7.

12 Gunthorpe MJ, Hannan SL, Smart D, Jerman JC, Arpino S et al. Characterization of SB-705498, a potent and selective vanilloid receptor-1 (VR1/ TRPV1) antagonist that inhibits the capsaicin-, acid-, and heat-mediated activation of the receptor. I Pharmacol Exp Ther 2007; 321: 1183-92.

13 Chizh BA, O’Donnell MB, Napolitano A, Wang J, Brooke AC et al. The effects of the TRPV1 antagonist SB-705498 on TRPV1 receptor-mediated activity and inflammatory hyperalgesia in humans. Pain 2007; 132: 132-41.

14 Dehpour AR, Samini M, Ghafourifar $\mathrm{P}$, Kyani H. Inhibition by lithium of neomycin-induced release of $\mathrm{N}$-acetyl-beta-glucosaminidase in the rat heart. Pharmacol Toxicol 1995; 76: 202-5.

15 Nayci A, Atis S, Ersoz G, Polat A. Gut decontamination prevents bronchoscopy-induced bacterial translocation. An experimental study in rats. Respiration 2004; 71: 66-71.

16 van denWijngaardRM, Welting $\mathrm{O}$, van derCoelenD, de JongeWJ, Boeckxstaens GE. Delayed visceral hypersensitivity in maternal separation depends on mast cell degranulation and is mediated by NGF and the nociceptor TRPV-1. Gastroenterology 2005; 128(Suppl. 2): A-125.

17 Meaney MJ. Maternal care, gene expression, and the transmission of individual differences in stress reactivity across generations. Annu Rev Neurosci 2001; 24: 1161-92.

18 Dobrev D, Ravens U. Therapeutically relevant concentrations of neomycin selectively inhibit P-type $\mathrm{Ca} 2^{+}$ channels in rat striatum. Eur I Pharmacol 2003; 461: 105-11.

19 Zhou Y, Zhao ZQ. Effects of neomycin on high-threshold $\mathrm{Ca}\left(2^{+}\right)$ currents and tetrodotoxin-resistant $\mathrm{Na}(+)$ currents in rat dorsal root ganglion neuron. Eur I Pharmacol 2002; 450: 29-35.

20 Beyak MJ, Ramji N, Krol KM, Kawaja MD, Vanner SJ. Two TTX-resistant $\mathrm{Na}+$ currents in mouse colonic dorsal root ganglia neurons and their role in colitis-induced hyperexcitability. Am I Physiol Gastrointest Liver Physiol 2004; 287: G845-55.
21 Laird JM, Souslova V, Wood JN, Cervero F. Deficits in visceral pain and referred hyperalgesia in Nav1.8 (SNS/PN3)-null mice. I Neurosci 2002; 22: 8352-6.

22 Drossman DA, Camilleri M, Mayer EA, Whitehead WE. AGA technical review on irritable bowel syndrome. Gastroenterology 2002; 123: 2108-31.

23 Lee HR, Pimentel M. Bacteria and irritable bowel syndrome: the evidence for small intestinal bacterial overgrowth. Curr Gastroenterol Rep 2006; 8: 305-11.

24 Pimentel M, Chow EJ, Lin HC. Normalization of lactulose breath testing correlates with symptom improvement in irritable bowel syndrome. A double-blind, randomized, placebocontrolled study. Am I Gastroenterol 2003; 98: 412-9.

25 Akbar A, Yiangou Y, Facer P, Walters $\mathrm{JR}$, Anand $\mathrm{P}$ et al. Increased capsaicin receptor TRPV1-expressing sensory fibres in irritable bowel syndrome and their correlation with abdominal pain. Gut 2008; 57: 923-9.

26 Delahunty T, Hollander D. A comparison of intestinal permeability between humans and three common laboratory animals. Comp Biochem Physiol A 1987; 86: 565-7.

27 Camilleri $M$, Gorman H. Intestinal permeability and irritable bowel syndrome. Neurogastroenterol Motil 2007; 19: 545-52.

28 Piche T, Barbara G, Aubert P, Bruley des Varannes $\mathrm{S}$, Dainese $\mathrm{R}$ et al. Impaired intestinal barrier integrity in the colon of irritable bowel syndrome patients: involvement of soluble mediators. Gut 2008; 58: 196-201.

29 Breen KJ, Bryant RE, Levinson JD, Schenker S. Neomycin absorption in man. Studies of oral and enema administration and effect of intestinal ulceration. Ann Intern Med 1972; 76: 211-8.

30 van den Wijngaard RM, Welting $\mathrm{O}$, de Jonge W, Boeckxstaens GE. Neomycin inhibits TRPV1 mediated stress-induced visceral hypersensitivity in maternal separated rats. Neurogastroenterol Motil 2006; 18: 735-6.

31 van den Wijngaard RM, Welting $\mathrm{O}$, Bulmer DC, Lee K, de Jonge WJ et al. Possible role for TRPV1 in neomycininduced inhibition of visceral hypersensitivity in rat. Neurogastroenterol Motil 2008; 20: 123. 